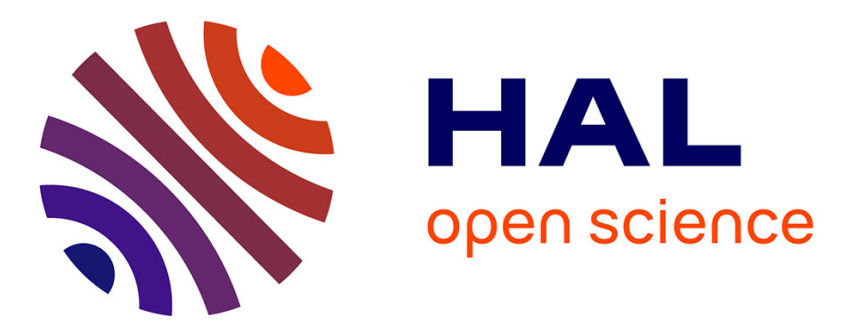

\title{
Impact of RF Front-end non-idealities on performances of SIMO-OFDM receiver
}

Xin Xu, Abhimanyu Sahai, Pierre-Francois Morlat, Jacques Verdier, Guillaume Villemaud, Jean-Marie Gorce

\section{- To cite this version:}

Xin Xu, Abhimanyu Sahai, Pierre-Francois Morlat, Jacques Verdier, Guillaume Villemaud, et al.. Impact of RF Front-end non-idealities on performances of SIMO-OFDM receiver. European Conference on Antennas and Propagation, Nov 2006, Nice, France. inria-00412071

\section{HAL Id: inria-00412071 \\ https://hal.inria.fr/inria-00412071}

Submitted on 27 Nov 2009

HAL is a multi-disciplinary open access archive for the deposit and dissemination of scientific research documents, whether they are published or not. The documents may come from teaching and research institutions in France or abroad, or from public or private research centers.
L'archive ouverte pluridisciplinaire HAL, est destinée au dépôt et à la diffusion de documents scientifiques de niveau recherche, publiés ou non, émanant des établissements d'enseignement et de recherche français ou étrangers, des laboratoires publics ou privés. 


\title{
IMPACT OF RF FRONT-END NON-IDEALITIES ON PERFORMANCES OF SIMO-OFDM RECEIVER
}

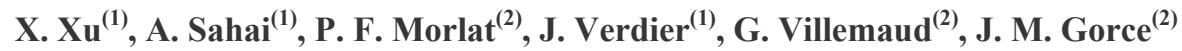 \\ ${ }^{(1)}$ LPM INSA-Lyon, 7 avenue Jean Capelle 69100 Villeurbanne FRANCE, Email:jacques.verdier@insa-lyon.fr \\ ${ }^{(2)}$ CITI INSA-Lyon, 21 avenue Jean Capelle 69100 Villeurbanne FRANCE, Email:pierre-francois.morlat@insa-lyon.fr
}

\section{Abstract}

Today, in OFDM systems, zero-IF receivers are very appealing, because they avoid costly IF filters. However, this very popular architecture implies IQ demodulation at RF and thus introduces new drawbacks and large performance degradations. It's therefore very important to study the impact of RF front-end nonidealities on performances of OFDM receiver. Especially, it's necessary to investigate the influence of I/Q gain and phase imbalance, local oscillator phase noise and non-linear distortion. In this paper, $802.11 \mathrm{~g}$ standard has been studied in terms of Bit Error Rate (BER) and constellation of each subcarrier. Simulated and measured results have been studied in the four arms SMI (Sample Matrix Inversion) as compared with a single antenna receiver. Two channel conditions, AWGN channel and multipath channel (taking account the fading correlation), have been modelled and studied to quantify WLAN performance degradations but also to evaluate the efficiency of a multi element antennas system at the receiver to improve significantly the performances of a wireless system. Advanced Design System (ADS - Agilent Technologies) software and a radio test-bed have been used in this work.

\section{INTRODUCTION}

In many contributions related to Wireless LocalArea-Network (WLAN), the spatial diversity is generally used at the receiver side. This class of systems is often named single-input multiple-output (SIMO) and the combination of SIMO architectures with the multicarrier technique orthogonal frequency division multiplexing is very promising [1]. Nevertheless, research has mainly focussed on systems impaired by additive white Gaussian noise (AWGN) and really, such analyses are not suffice. The influence of non-perfect oscillators, non-perfect power or low noise amplifier and I/Q gain and phase imbalance on the performance of SIMO-OFDM system can be very critical and must be studied exactly [2][3]. Moreover, the impact of RF impairments on a receiver depends on RF front-end topologies [3]. Direct conversion is an alternative wireless receiver architecture to the well-established superheterodyne, particularly for highly integrated, low power terminals. Consequently, it's very important to analyse and to quantify the influence of RF impairments in SIMO architecture versus front-end architecture. In this paper, a complete analysis of WLAN 802.11g standard is reported in terms of Bit Error Rate (BER) especially. At first, (section II) we present our global system evaluation approach based on the use of a $2 \times 2$ MIMO radio plateform connected to the Advanced Design System (ADS) [4]. In part III, we discuss the key points of RF front-end limitations which strongly decrease receiver efficiency. The Sample Matrix Inversion (SMI) smart antennas algorithm is used for simulations and two types of channel model, AWGN and multi-path models, have been investigated. The section III.1 deals with IQ imbalance impact. Some interesting results for joint compensation of fading effects are presented. In sections III.2 and III.3, the influence of the power amplifier non-linearity and local oscillator phase noise is reported. The $1 \mathrm{~dB}$ compression point and the third order intercept point are modelled to quantify the intermodulation distortion characteristics of each system and the slope and the level of phase noise spectrum related to several oscillator phase noise models are discussed in detail.

\section{Global SySTEM Evaluation APPROACH}

The radio platform presented in Figure 1 is an analysis tool which allows testing the most advanced techniques in conception, modelling and processing for radiocommunication systems. This platform is composed of high technology equipments developed by Agilent Technologies: the ADS software, and measurement hardware which are two arbitrary waveform generators (ESG4438C) and a vector spectrum analyzer (VSA89641) with two RF inputs [4].

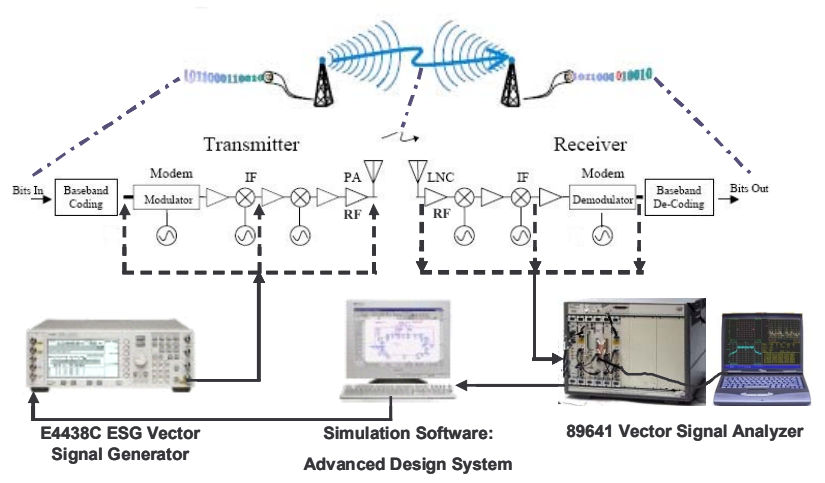

Figure 1 : The radio platform structure

It allows measurements up to $6 \mathrm{GHz}$, with a received bandwidth analysis of $36 \mathrm{MHz}$. The arbitrary waveform generator by itself is able to generate any complex 
signal which is then possible to analyse after propagation with the vector spectrum analyzer. The vectorial analysis software can demodulate this signal and offers visualization options to show spectrum, constellations, BER, EVM... in order to accurately estimate the transmission system quality.

With this connected solution a software/hardware interaction allows us to conceive and test very complex and realistic systems. Indeed the modelled signal with ADS can be loaded in the internal memory of the ESG, and then the signal measured by the VSA can be recorded and transferred to the software. This interaction can take place at any part of the chain (baseband, IF or RF frequency). ADS permits to model complete system, from the device to the whole architecture. With Ptolemy tool, analogical and digital signal live together, it allows us to study all the fields bound to telecommunications by making them act mutually. We can therefore estimate the impact of the different RF impairments of the front end (DC offset, phase noise, distortion, IQ imbalance), and also the propagation environment (AWGN, Fading...). Any component toolbox (receiver, transmitter, channel) are customizable and models more or less elaborated can be add. A very realistic modelling of the system can be simulated taking into account the characteristic of the channel but also the physical and technological properties of the front end. For instance, some parts of the standard receiver and transmitter can be replaced by specific devices (such as VCO, mixer, Power amplifier or LNA) with appropriate characteristics to evaluate real performances.

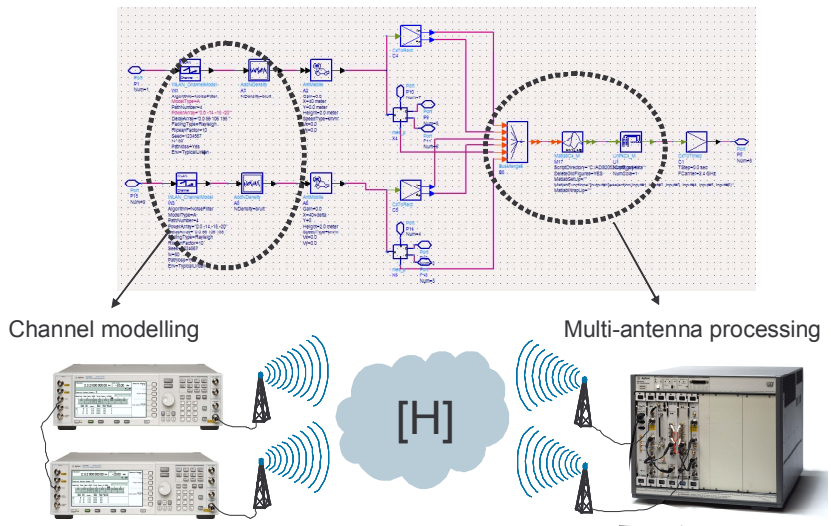

Figure 2 : Example of a $2 \times 2$ connected solution between ADS and hardware measurements

Finally, a great interest of this platform is to permit the validation of channel models with direct comparison with signal recorded by the VSA in any typical environment. With the two RF inputs of the VSA it is also possible to analyze and evaluate the performance of SIMO or MIMO systems or to characterize multichannel models (Figure 2). By the use of two synchronized generators, a 2 by 2 complete system can be directly measured, easily extended to NxM systems by switching principles. Figure 3 shows the example of the received spectrum on the four arms of a multiple antenna system with spatial diversity, revealing the impact of multipath fading channels. We are then currently studying the implementation of a $802.11 \mathrm{n}$ scheme based on this $2 \times 2$ structure.
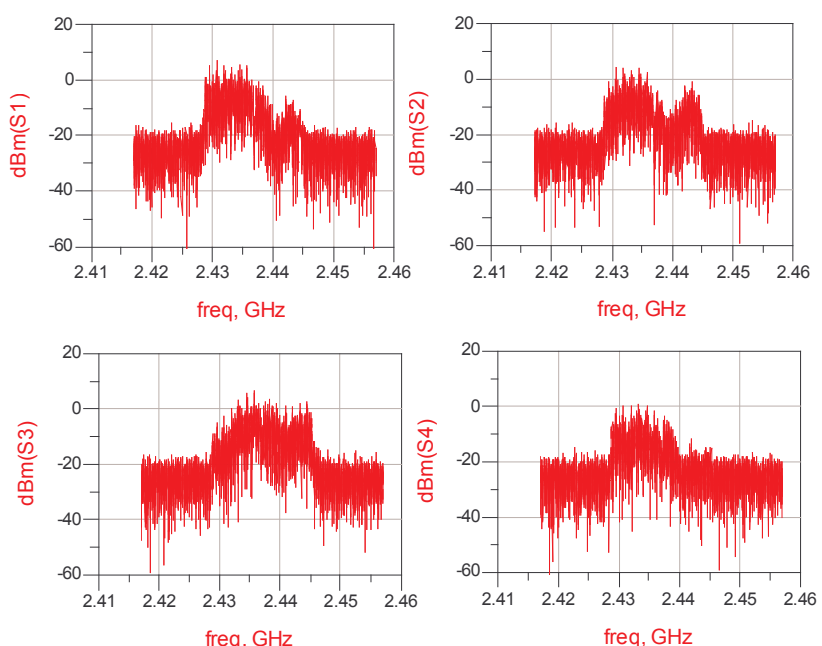

Figure 3: Received spectrum on four spatially decorrelated channels in hard multipath environment

\section{IMPACT OF RF IMPAIRMENTS ON PERFORMANCES OF FOUR ANTENNAS 802.11G RECEIVER}

Our objective is to simulate a really close to reality transmission system and to have a better estimation of the advantage of the Sample Matrix Inversion (SMI) smart antennas algorithm [5]. A complete 802.11g transmission system was modelled with ADS software, versus different types of channel (average white Gaussian noise or various multipath models). All further presented results are based on the following configuration : the BER performances of the four arms SMI receiver compared to a single antenna receiver with a $36 \mathrm{Mbps}$ transmission rate (16QAM). To simulate the multipath fading, we use typical office environment properties based on ETSI channel models [6].

\section{III.1 IQ imbalance effect}

An important issue in a direct conversion receiver is IQ imbalance [3][7]. A non-ideal I\&Q downconversion may result if the phase and amplitude of the branches are not matched and cause a warping of received signal constellation diagram. Generally, IQ imbalance is characterized by two parameters: gain imbalance (or amplitude mismatch) and phase orthogonality mismatch. In ADS, this effect is modelled directly in the form of the parameters 'Gain Imbalance' and 'Phase Imbalance' of the QAM demodulator block. We studied the impact of IQ imbalance in AWGN channel (an example of 16QAM constellation is presented in figure 4 ; the increased error due to IQ imbalances 
clearly appears in this figure.) and multipath channel but we present only some results in multipath environment, in this section.
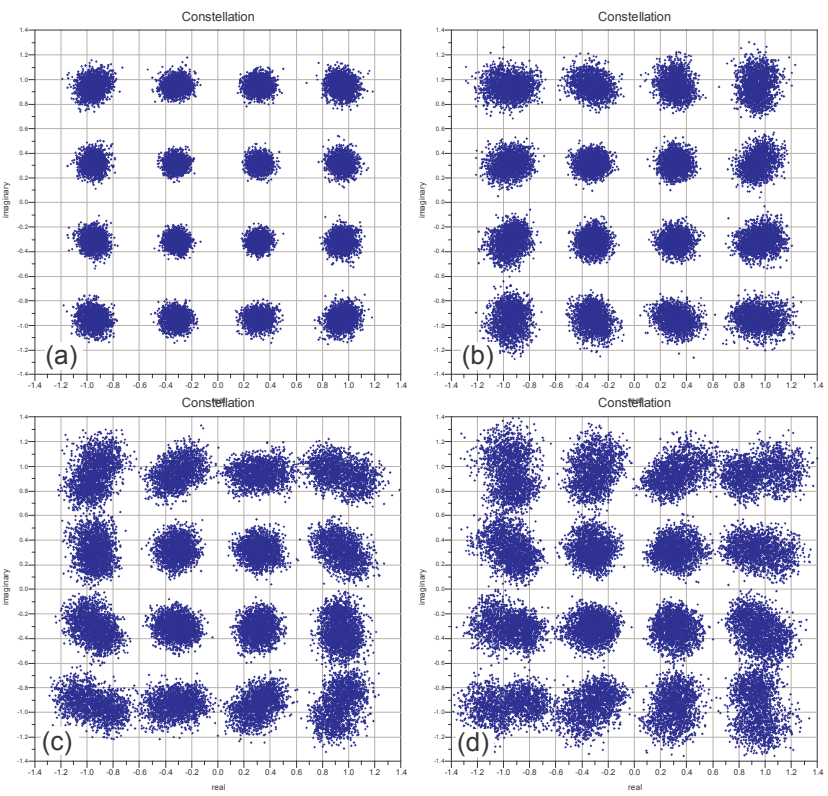

Figure 4 : Influence of IQ imbalance on a 16QAM constellation in AWGN channel (a) without imbalance, (b) $1 \mathrm{~dB}$ gain imbalance, (c) $10^{\circ}$ phase imbalance, (d) $1 \mathrm{~dB}$ gain and $10^{\circ}$ phase imbalance

A first evaluation of the SMI gain is presented on figure 5. This result is obtained for an IQ gain imbalance varying from 0 to $1 \mathrm{~dB}$. The single antenna case was realised for a $10 \mathrm{~dB}$ signal-to-noise ratio and the four antennas case with a $6 \mathrm{~dB}$ one, in order to obtain a comparable range of BER (around $10^{-3}$ for zero gain imbalance). For each point, 1000 frames of 100 bytes were simulated. Results are plotted in relative BER (zero gain imbalance BER value taken for reference). It clearly appears that the SMI algorithm efficiently compensate RF impact and, as expected we obtained an important increase of the relative BER for a single antenna compared to the AWGN case.

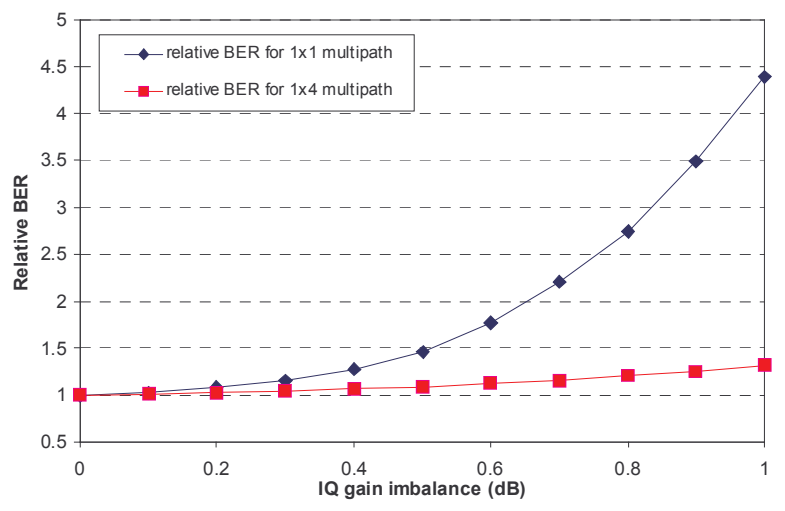

Figure 5 : Relative BER vs. IQ gain imbalance in multipath channel

The same approach was used to evaluate the improvement in presence of phase imbalance from 0 to
10 degrees. A great improvement is also obtained as shown in Figure 6. However, it is important to note that those results would be degraded if we consider more correlated channels.

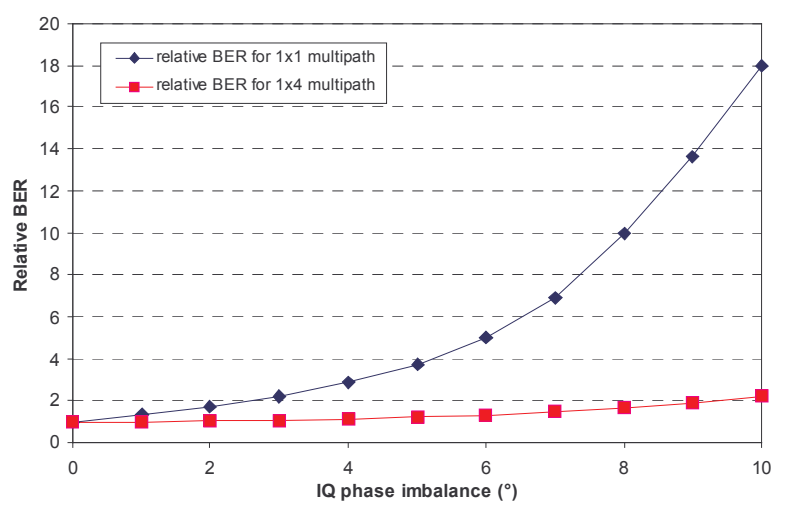

Figure 6 : Relative BER vs. IQ phase imbalance in multipath channel

In addition, some none uniform gain imbalances on the four arms of the receiver have been studied. For independent random values from -1 to $1 \mathrm{~dB}$ we obtain a quite stable compensation: starting from a zero gain imbalance BER of $1.9 .10^{-3}$, a $1.99 .10^{-3}$ mean BER is performed with a standard deviation of $1.2 \cdot 10^{-4}$.

\section{III.2 Oscillator phase noise effect}

The influence of frequency synthesizer phase noise on the performance of a SIMO-OFDM system is a very important characteristic. Generally, in single-input single output (SISO) OFDM systems, the effect can be split into a multiplicative part, which is equal for all subcarriers and therefore referred to as common phase error, and an additive part, which is referred to as intercarrier interference [8][9].

The standard model for phase noise in free-running oscillator [10][11] is given in terms of the slope of the phase noise spectrum in a Bode diagram. It considers the phase noise as a superposition of several components (Wiener frequency noise, Flicker frequency noise, Wiener phase noise, Flicker phase noise and white phase noise) with different spectrum signature. This model shows great coherence with measurments.

In ADS, phase noise can be modelled directly using the parameter "PhaseNoise Data" of the local oscillator block. It contains double values of offset frequency $(\mathrm{Hz})$ and single sideband relative power level $(\mathrm{dBc} / \mathrm{Hz})$. Interpolation is applied between these frequency domain points as needed to give a full time domain simulation definition for this phase noise.

We studied and compared the performance of the SISO and SIMO-OFDM systems assuming a pure AWGN channel. Figure 7 shows the BER vs. SNR curves obtained for both systems assuming the Flicker noise model, with three different phase noise characteristics :

PN1: Noiseless local oscillator 
PN2: $-60 \mathrm{dBc} / \mathrm{Hz}$ at $100 \mathrm{~Hz}$ offset

$-90 \mathrm{dBc} / \mathrm{Hz}$ at $1 \mathrm{KHz}$ offset

$-150 \mathrm{dBc} / \mathrm{Hz}$ at $100 \mathrm{KHz}$ offset

\section{PN3: $-20 \mathrm{dBc} / \mathrm{Hz}$ at $100 \mathrm{~Hz}$ offset $-50 \mathrm{dBc} / \mathrm{Hz}$ at $1 \mathrm{KHz}$ offset $-110 \mathrm{dBc} / \mathrm{Hz}$ at $100 \mathrm{KHz}$ offset}

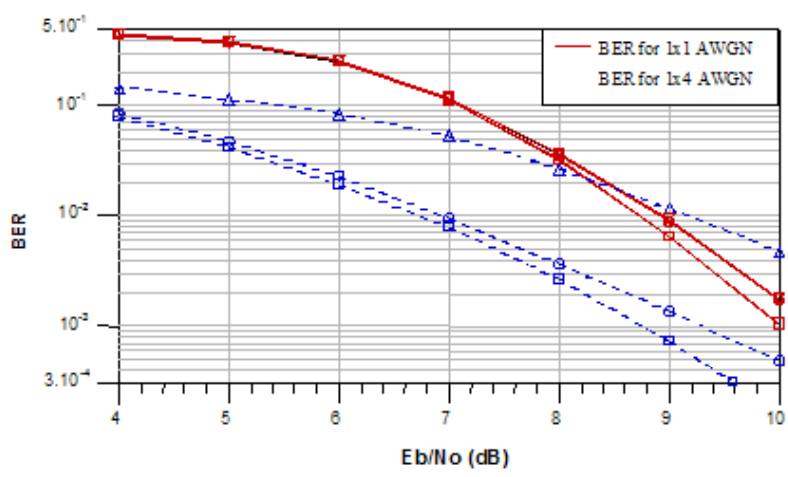

Figure 7 : BER vs. Eb/No in AWGN channel

As shown in Figure 7, the phase noise greatly degrades the BER in the case of the SISO system, but the SIMO arrangement does not seem to be able to compensate for its ill-effects. In Figure 8, the simulated data are plotted in relative BER. It clearly appears that the SMI algorithm doesn't compensate the increases of the local oscillator phase noise. Other results have to be obtained to clearly show the limitation of this technique.

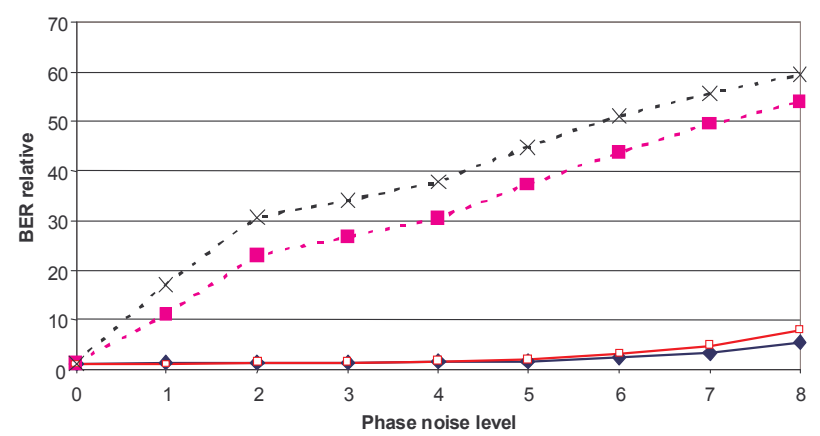

$\longrightarrow-1$ X1 - AWGN - - - 1 X4 - AWGN $\square-1 \times 1$ - Multipath $\ldots-x-1$ - 1 4 - Multipath

Figure 7 : relative BER vs. Phase noise level in AWGN channel and multipath channel

(“0” no phase noise noise ; " 8 " $-40 \mathrm{dBc} / \mathrm{Hz}$ at $100 \mathrm{~Hz}$ offset)

\section{III.3 Influence of power amplifier non-linearity}

It's very interesting to study the effects of harmonic [12] distortion and intermodulation distortion. Indeed, a linear PA is needed in the case of non-constant envelope signals. The linearity of a PA is a key parameter as it is closely related to power consumption and to distorsion, hence BER. All gain compression characteristics are modelled (in ADS software) using a polynomial expression up to the saturation.

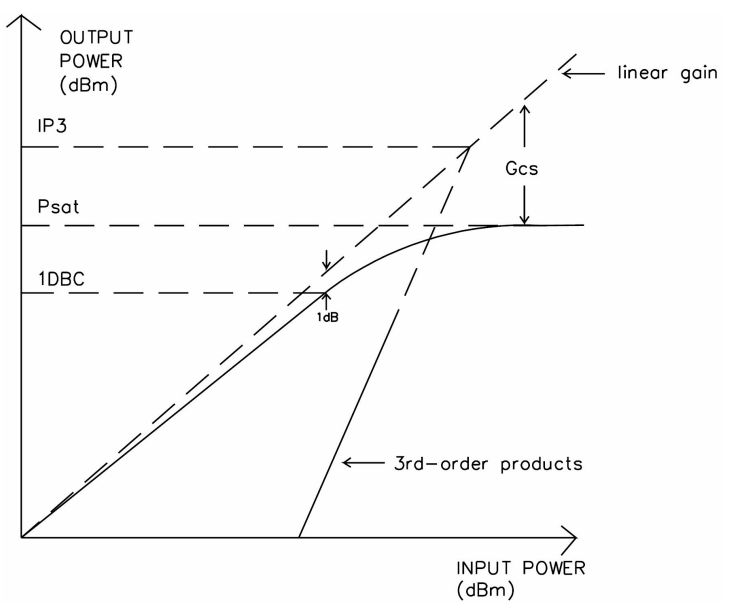

Figure 9: Non-linear element characterisation for non-linear models

Figure 9 shows characterisation parameters for nonlinear elements. In Figure 10, the effects of $1-\mathrm{dB}$ compression point and the intermodulation intercept point (IP) associated to third-order products on BER performances (for $\mathrm{Eb} / \mathrm{No}=10 \mathrm{~dB}$ ) are reported for multipath channel. Compared with the case of AWGN channel (not reported), the SMI gain seems to be very interesting.

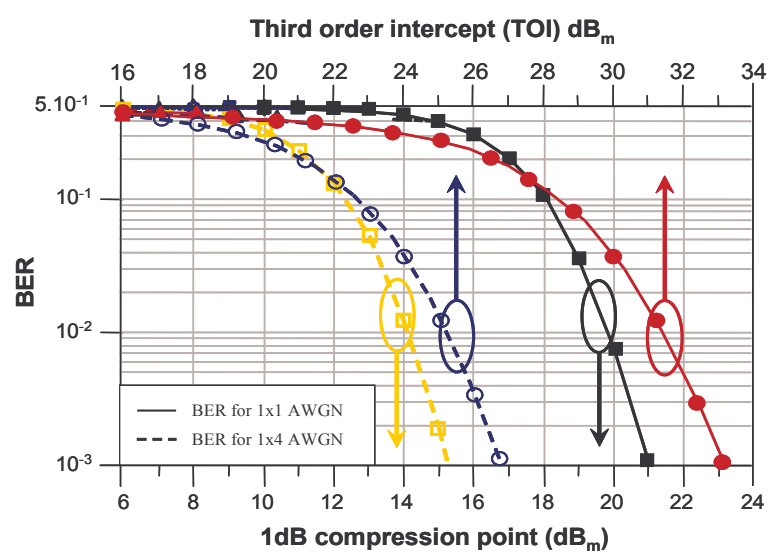

Figure 10: BER vs. TOI and $1 \mathrm{~dB}$ compression point in multi-path channel (linear gain of PA is $10 \mathrm{~dB}$ and $\mathrm{Eb} / \mathrm{No}=10 \mathrm{~dB}$ )

\section{Conclusion}

Today, a global approach combining multiple antennas, OFDM technique and SDR principles appears really a very good choice particularly for future broadband wireless systems. Unfortunately, this combination is not so evident: multiple antenna algorithms and SDR are resource-expensive and OFDM technique is sensitive to RF front-end performances.

We have presented here a system approach to simulate and measure complete transmission scheme taking into account all parts of the structure, allowing a 
realistic link budget. Thus, the influence of RF impairments could be evaluated, depending on architecture choices, SIMO algorithm and channel conditions.

First simulated and measured results based on a $1 \times 4$ antennas transmission with a $802.11 \mathrm{~g}$ receiver have been discussed depending on the environment, and then the impact of IQ imbalance, oscillator phase noise and power amplifier non-linearity have been studied in details.

Therefore we have here the opportunity for a complete study of all RF impairments consequences, and we precisely want to focus on the potential of large bandwidth acquisition approach for reconfigurable terminals, multiple antennas allowing interference cancellation and multi-channel capabilities.

\section{REFERENCES}

[1] P. Rykaczewski, D. Pienkowski and R. Circa, "Signal Path Optimization in Software-Defined Radio Systems" IEEE Trans. On Mic. Th. And Tech., vol. 53, No 3, 2005

[2] S. Woo et al., "Combined Effects of RF Impairments in the Future IEEE 802.11n WLAN Systems", IEEE Vehicular Technology Conference, Spring 2005, Vol. 2, May 2005

[3] M. Brandolini, P. Rossi, D. Manstretta and F. Svelto, "Toward Multistandard Mobile Terminals - Fully Integrated Receivers Requirements and Architectures", IEEE Trans. on Mic. Th. And Tech., vol. 53, No 3, 2005

[4] http://eesof.tm.agilent.com/products/ads_main.html

[5] I. J. Gupta, "SMI adaptive antenna arrays for weak interfering signals”, IEEE Trans. Antennas and Propagat., vol. 34, pp $1237-1242,1986$

[6] Channel Models for HIPERLAN/2 in different indoor Scenarios. ETSI EP BRAN 3ER1085B, March 1998

[7] J. Tubbax et al., "Joint Compensation of IQ Imbalance, Frequency Offset and Phase Noise in OFDM Receivers", European Trans. On Telecom., S. Issue on Multi-Carrier Spread-Spectrum, vol. 15, 2004

[8] C. Muschallik, "Influence of RF oscillators on an OFDM signal", IEEE Trans. On Consumer Electronics, Vol.41, No3, pp.592-603, August 95

[9] S. Wu and Y. Bar-Ness, "Performance analysis on the effect of phase noise in OFDM systems," in Proc. IEEE Seventh International Symposium on Spread Spectrum Techniques and Applications, vol. 1, pp.133-138, 2002

[10] T. E. Parker, "Characteristics and sources of phase nois in stable oscillators", Proc. $41^{\text {st }}$ Annual Frequency Control Symposium, 1987

[11] B. Razavi "Phase locking in high performance systems : From Devices to Architectures" publisher J. Wiley \& Sons Inc, 736 pages, 2003

[12] F.H. Gregorio and T.I. Laakso, "The performance of OFDMSDMA systems with power amplifier non-linearities" in Proc. 2005 Finnish Signal Processing Symposium, pp. 58-61, 2005 